\title{
INTEGRACIÓN DE LAS TIC EN LA ENSEÑANZA DE LA HISTORIA EN EDUCACIÓN MEDIA SUPERIOR
}

\section{INTEGRATION OF ICT IN THE TEACHING OF HISTORY IN HIGHER SECONDARY EDUCATION}

\author{
Lic. Oscar David Bustos Torres ${ }^{11}$ \\ Dra. Karla Lariza Parra Encinas ${ }^{22}$ \\ Universidad Autónoma de Baja California
}

\section{RESUMEN:}

Las Tecnologías de la Información y la Comunicación se han convertido en el elemento esencial de nuevos contextos y espacios sociales. Las instituciones educativas se encuentran analizando las constantes

$1 \quad{ }^{1}$ Licenciado en Historia. Maestrante en Educación en la Facultad de Ciencias Humanas, de la Universidad Autónoma de Baja California. Orcid ID: https://orcid.org/00000001-7173-2231

$2 \quad 2$ Maestra en Tecnologías para el Aprendizaje. Doctora en Educación. Profesora Investigadora de la Licenciatura en Ciencias de la Educación, en la Universidad Autónoma de Baja California. Orcid ID: https://orcid.org/00000002-2855-2224.

Artículos, entre otros: Parra, Karla L. (2017) "CLAVE PARA EL ÉXITO, EN LA ALFABETIZACIÓN DIGITAL DE LOS ADULTOS MAYORES" del libro: "Experiencias en el uso de tecnologías en los procesos educativos”, Editorial Redipe. transformaciones derivadas de la incorporación de las tecnologías en los contextos educativos actuales. Para lograr particularmente el interés de estudiantes de bachillerato en asignaturas como "Historia", es necesario que el desarrollo de las clases y los programas educativos sean atractivos para ellos.

En el presente artículo, se comparte un estudio cuantitativo realizado con los docentes de Historia de una institución de educación media superior pública mexicana, basado en un instrumento que analiza las competencias digitales básicas, clasificadas en siete dimensiones. 
Los resultados del estudio muestran como áreas de oportunidad para los docentes, su formación en competencias digitales básicas para lograr crear, publicar y compartir material didáctico atractivo para sus estudiantes.

\section{PALABRAS CLAVE:}

Competencias digitales; rol docente; actualización docente; enseñanza de la historia.

\section{ABSTRACT:}

Abstract: Information and Communication Technology has become an essential element for new contexts and social spaces.

Educational institutes are analyzing the constant transformation due to the incorporation of technology. Specifically, to achieve interest of high school students in subjects such as \&quot;History\&quot; it is necessary to incorporate and integrate such technology to achieve interest in the subject.

In this article, we share a quantitative study conducted with history educators in a

Mexican high school. A method that has been developed to analyze the use of digital methods in seven specific areas.

The results of the study reveal areas where educators need to grow; their training in basic digital skills, ability to create, to publish and share educational material and

importantly make learning more attractive to students.

KEYWORDS: Digital competency; teaching role; teacher update; teaching history.

\section{INTRODUCCIÓN}

La irrupción inminente de las Tecnologías de la Información y la Comunicación (TIC) en diversos sectores de la sociedad ha propiciado notables cambios en la educación, lo que ha provocado la presencia de herramientas y tecnologías nuevas en las instituciones educativas en general, las aulas y en los procesos de aprendizaje en particular.

Lo anterior ha originado inevitables modificaciones en los "roles" de estudiantes y profesores, al verse alterada la dinámica tradicional de ambos, así como su interacción y naturales medios de comunicación. De acuerdo con Cabero (2007) estos nuevos escenarios "requieren de una reflexión hacia el uso e incorporación de las tecnologías, los contextos educativos actuales deberán apostar por una integración crítica, en la cual se defina el qué, por qué y para qué de su incorporación y aprovechamiento" (p. 5).

Enfocarse en la incorporación de las tecnologías solo por hacerlo, o evitar el análisis crítico y reflexivo antes de realizarla, es un error. Dicha integración de las TIC deberá entenderse como enriquecimiento de la práctica educativa.

La Organización de las Naciones Unidas para la Educación, la Ciencia y la Cultura (UNESCO) ha desarrollado programas que involucran estándares, relacionados específicamente con las competencias docentes. Tal es el caso de los Estándares de Competencias en TIC para docentes (2008), que apunta, en general, a mejorar la práctica de los docentes en todas las áreas de su desempeño profesional, combinando las competencias en TIC con innovaciones en la pedagogía, el plan de estudios (currículo) y la organización escolar; aunado al propósito de "lograr que los docentes utilicen competencias en TIC y recursos para mejorar sus estrategias de enseñanza, cooperar con sus colegas y, en última instancia, poder convertirse en líderes de la innovación dentro de sus respectivas instituciones" (p.4).

En México, por medio del Plan Nacional de Desarrollo 2013-2018 (Gobierno de la República, 2012), se declara el compromiso 
de búsqueda de la calidad educativa, con el desarrollo de un sistema de vanguardia en la educación media superior. La Reforma Integral de Educación Media Superior (RIEMS) 2008, que tiene la intención de contribuir a la resolución de los principales problemas de la educación media superior de nuestro país, así como responder a las demandas de la dinámica mundial (Secretaría de Educación Pública, 2008); establece especial atención al desarrollo de competencias docentes, mismas que "representan una capacidad para movilizar varios recursos cognitivos para hacer frente a un tipo de situaciones" (Perrenoud, 2007, citado en Guzmán \& Marín, 2011, p.154), esto es, que posibilite al docentelasolución de problemas.

Muchos autores han investigado sobre el desarrollo de competencias docentes resaltando particularmente, que las competencias digitales modifican las prácticas y potencian el aprendizaje. (Ortega, M. y Reyes, O., 2012; Salinas, J., Benito, B. y Lizana, A. 2014; Cabero, J. 2010; Reig, D. 2019, y Díaz-Maroto, I. y Cascales, A. 2015).

Por otro lado, particularmente en lo correspondiente al abordaje de la Historia, Florescano (1999) señala que su enseñanza "es indispensable para el conocimiento del ser humano viviendo en sociedad. En México se ha olvidado este propósito. De hecho, la enseñanza de la historia es espejo del desastre mayor que padece el sistema educativo nacional" (p. 22)

Vélez y Badillo (2017) se refieren a la problemática de la enseñanza docente de la Historia, sosteniendo que los docentes acuden a sus aulas poniendo en práctica lo que entienden por enseñanza de la historia, que en ocasiones se asemeja a "la forma como ellos mismos aprendieron y que continúa instruyéndose en los centros escolares, sin resignificar los productos históricos mediante una construcción constante y cambiante a partir de lo vivido" (p.3).
Comúnmente se aprende Historia memorizando textos y fechas, Casamayor (2010) señala que "no basta con memorizar un dato o conocer un proceso, conviene saber utilizarlo", por lo tanto, podemos decir que hemos aprendido algo nuevo cuando somos capaces de matizarlo, de solucionar nuevos y diferentes problemas ( $p$. 54).

Se vuelve entonces necesario sensibilizar a profesores e instituciones educativas en general, respecto a la integración de las TIC dentro de las aulas, despertando el interés y gusto por la Historia, ya que los docentes frente a grupo deben advertir que la enseñanza requiere un cambio en la forma de abordar los contenidos.

De acuerdo con Miguel Irigoyen (2014) "la aportación de recursos TIC en la asignatura de Historia aumenta considerablemente la motivación de los alumnos, desarrollando competencias no solo del ámbito digital, sino también del social y científico" (p. 42).

En sintonía con Hernández, R., Bautista, D.E., y Hernández, J.J. (2015), "El uso de la tecnología impone diversos retos al razonamiento de las y los educandos y los lleva a desarrollar habilidades mentales y procedimientos que de una u otra manera, constituyen un aporte para su formación" (p. 1025).

Para efectos del presente estudio se adoptan los trabajos realizados por la estadounidense María H. Andersen (2009), quien propone un listado de competencias digitales con diversas clasificaciones basadas en subcompetencias en su investigación "Teaching and Learning in the Digital Age".

Lo anterior derivado de la experiencia en la incorporación de nuevas tecnologías y sus usos productivos (respecto a aprendizaje o desarrollo profesional) en las aulas. Andersen sostiene que estas siete serían las competencias globales que todo universitario 
debe poseer, por lo tanto, todo profesor de cualquier nivel educativo debe desarrollarlas.

\section{ABORDAJE METODOLÓGICO}

La investigación se realizó desde un enfoque cuantitativo, ubicada en la modalidad de investigación descriptiva, que consiste en la caracterización de un fenómeno con el fin de establecer su comportamiento (Arias, 2004); de acuerdo al momento de recogida de la información es de corte transversal, cuyos datos provienen directamente de la realidad (Sabino,2002).

Fue utilizado un instrumento escala tipo Likert, conformado por 61 afirmaciones, reactivos distribuidos en siete categorías (dimensiones teóricas) cada una correspondiente a una de las competencias digitales clasificadas por Andersen (2009):

- Habilidades básicas en la web

- Organización
- Comunicación

- Buscar y gestionar información

- Privacidad, seguridad y ley

- Presentación

- Modos de aprender

La escala tipo Likert, trata de un método desarrollado por Rensis Likert a principios de los treinta; sin embargo, se trata de un enfoque vigente y bastante popularizado. Consiste en un conjunto de ítems presentados en forma de afirmaciones o juicios ante los cuales se pide la reacción de los sujetos a los que se les administra.

Es decir, se presenta cada afirmación y se pide al sujeto que externe su reacción eligiendo uno de los cinco puntos de la escala. A cada punto se le asigna un valor numérico. Así, el sujeto obtiene una puntuación respecto a la afirmación y al final se obtiene su puntuación total sumando las puntuaciones obtenidas en relación a todas las afirmaciones (Hernández, R., Fernández, C. y Baptista, P., 2006).

Se operacionalizaron las variables, con su definición conceptual y operacional de cada una de ellas (Tabla 1):

\begin{tabular}{|c|c|}
\hline $\begin{array}{l}\text { Factores (Dimensiones } \\
\text { Teóricas) }\end{array}$ & Descripción \\
\hline Habilidades básicas en la web & $\begin{array}{l}\text { Presenta } 13 \text { sub-competencias y consiste en el desarrollo de habilidades básicas de conexión } \\
\text { a Internet. Por ejemplo: hacer y compartir un video. }\end{array}$ \\
\hline Organización & $\begin{array}{l}\text { Se integra por } 4 \text { sub-competencias y se refiere a las habilidades para organizarse con el uso de } \\
\text { herramientas digitales. Por ejemplo: configurar un calendario. }\end{array}$ \\
\hline Comunicación & $\begin{array}{l}\text { Se integra por } 11 \text { sub-competencias, se refiere a la habilidad para comunicarnos haciendo uso } \\
\text { de herramientas digitales y electrónicas. Por ejemplo: gestionar el correo electrónico. }\end{array}$ \\
\hline Buscar y gestionar información & $\begin{array}{l}\text { Se compone de } 10 \text { sub-competencias, consiste en la habilidad de investigar en Internet, } \\
\text { localizar documentos, imágenes e información diversa. Por ejemplo: usar marcadores basados } \\
\text { en la web. }\end{array}$ \\
\hline Privacidad, seguridad y ley & $\begin{array}{l}\text { Presenta } 7 \text { sub-competencias, se trata de la habilidad para conocer las cuestiones de seguridad } \\
\text { que hay que tener en cuenta al utilizar Internet. Por ejemplo: saber qué uso podemos dar a } \\
\text { materiales que encontramos en la red. }\end{array}$ \\
\hline Presentación & $\begin{array}{l}\text { Se integra por } 11 \text { sub-competencias, trata de la habilidad para presentar información de forma } \\
\text { electrónica, audiovisual, etc. Por ejemplo: determinar la audiencia y la apropiada longitud de las } \\
\text { presentaciones. }\end{array}$ \\
\hline Modos de aprender & $\begin{array}{l}\text { Se conforma por } 5 \text { sub-competencias, Consiste en la habilidad para utilizar las herramientas } \\
\text { digitales para aprender. Por ejemplo usar un blog para seguir el propio proceso de aprendizaje. }\end{array}$ \\
\hline
\end{tabular}

Tabla 1. Operacionalización de las variables, con su definición conceptual. 
Se optó por aplicar la encuesta de manera personalizada para el cumplimiento de la muestra de 45 profesores de nivel medio superior, que impartieran asignaturas de Historia y/o asignaturas a fines. Se acudió a dos planteles ambos turnos en repetidas ocasiones, durante una semana para abordar a los profesores y solicitar que respondieran la encuesta.

\section{RESULTADOS:}

En cuanto al Instrumento de competencias digitales, se presenta en la tabla 2, el coeficiente de fiabilidad obtenido en el tratamiento de las 7 dimensiones teóricas:

\begin{tabular}{|l|l|}
\hline \multicolumn{2}{|c|}{ Estadísticas de fiabilidad } \\
Alfa de Cronbach & $\mathrm{N}$ de elementos \\
\hline .917 & 7 \\
\hline
\end{tabular}

Tabla 2. Estadística de fiabilidad dimensiones teóricas

En cuanto a las estadísticas descriptivas y medias de los factores o dimensiones teóricas y la media general se aprecian los siguientes resultados:

\begin{tabular}{|l|l|l|l|l|}
\hline & $\mathrm{N}$ & Mínimo & Máximo & Media \\
\hline HBW & 45 & 1.00 & 4.15 & 2.3214 \\
\hline ORG & 45 & 1.00 & 4.00 & 1.7611 \\
\hline COM & 45 & 1.09 & 4.82 & 3.1006 \\
\hline BUS & 45 & 1.00 & 4.00 & 2.2822 \\
\hline PRIV & 45 & 1.00 & 5.00 & 2.4698 \\
\hline PRE & 45 & 1.00 & 3.48 & 1.5525 \\
\hline MOD & 45 & 1.00 & 4.80 & 2.0267 \\
\hline MEDGR & 45 & 1.15 & 4.10 & 2.2396 \\
\hline $\begin{array}{l}\text { N válido } \\
\text { (por } \\
\text { lista) }\end{array}$ & 45 & & & \\
\hline
\end{tabular}

Tabla 3. Estadísticas descriptivas (Medias) de dimensiones teóricas y media general.

Como se puede observar en la mayoría de los factores o dimensiones teóricas, se encuentran entre 2 "Poco competente" y 3 "Competente", siendo la variable o el factor "COMUNICACIÓN" el que se aprecia más elevado.

Lo anterior significa que los 45 profesores reconocen presentar más competencias digitales de comunicación. Esto es, utilizan el Internet y las tecnologías de la Información y la comunicación para "comunicarse". Utilizan el correo electrónico, utilizan redes sociales, etc.

Por otro lado, se refleja que las competencias de "Presentación y Organización" son las más bajas, esto es, no aprovechan las potencialidades de las TIC para la organización de sus actividades diarias y lo más relevante, para sus presentaciones en clase.

El concepto de relación o correlación entre dos variables se refiere al grado de parecido o variación conjunta existente entre las mismas. (ESF. s.f.) El coeficiente de correlación de Pearson, pensado para variables cuantitativas (escala mínima de intervalo), es un índice que mide el grado de covariación entre distintas variables relacionadas linealmente. Adviértase que "variables relacionadas linealmente" significa que puede haber variables fuertemente relacionadas, pero no de forma lineal, en cuyo caso no proceder a aplicarse la correlación de Pearson (Kovachi, s.f.)

Se analizaron los datos para detectar la existencia de correlaciones entre las competencias y subcompetencias analizadas y como resultado interesante se resalta que la correlación más alta es la existente entre la dimensión "Presentación" y "Modos de aprender" $(r=.882$ y $p=.000)$. Lo anterior responde a las habilidades para utilizar las herramientas digitales para aprender y al poco interés por comprender el uso de las TIC en educacioon media superior.

La discusión de los resultados se desarrolló teniendo en cuenta, que se requiere estudiar la influencia que ejerce la integración de las Tecnologías de la Información y la Comunicación (TIC) como estrategia para la enseñanza de la 
Historia en la Educación Media Superior.

Los resultados han sido muy interesantes, se detecta un nivel muy bajo de competencias digitales, el $93 \%$ del profesorado encuestado se reconoce "nada competente" en determinar la audiencia y la apropiada longitud de sus presentaciones (item 46); el $87 \%$ de los profesores señalan sentirse particularmente "poco competentes" en el diseño de buenas presentaciones para sus estudiantes (item 47); el $89 \%$, es decir, 40 profesores, desconocen en qué consiste una presentación no lineal, no logrando así, identificar lo que sería atractivo para sus estudiantes (item 50).

El total del profesorado se reconoce "nada competente" en la búsqueda de imágenes y audios de alta calidad para utilizarlos en presentaciones (con copyrights apropiados) y compartirlos en internet con sus estudiantes (items 52 y 53). Respecto a los modos de capturar un video en Internet (item 55), los 45 profesores señalaron sentirse "nada competente".

Como puede apreciarse, la habilidad para presentar información de forma electrónica y audiovisual dificilmente se encuentra presente en el profesorado abordado.

Por último, se resalta que en la dimensión "Organización", integrada por cuatro subcompetencias, misma que se refiere a las habilidades para organizarse con el uso de herramientas digitales (tales como la configuracion de un calendario); el $96 \%$ de los profesores se ha reconociddo "poco competente". Esto es, que unicamente dos profesores seleccionaron la opción "competente" en el item 14, referido a la configuración de calendarios y al uso de la gestión del tiempo con ayuda de TIC.

\section{CONCLUSIONES}

Despues del análisis detenido de los resultados se determina la fiabilidad del instrumento, lo que sostiene los trabajos de Andersen (2009), sobre la clasificación de competencias digitales que nos ofrece. Por un lado, y por el otro, al detecctar que el profesorado abordado carece de competencias digitales particularmente las relacionadas a la "presentación", enciende una alerta.

Lo anterior debido a que se espera que los profesores, en apoyo de las TIC, genere materiales didácticos atractivos para sus estudiantes. Cabe destacar que lo importante de las TIC a la hora de aplicarlas a la enseñanza de la Historia, como señala Miguel (2014) "no es tener los conocimientos de un informático, sino pensar y discurrir acerca de cómo estas herramientas pueden ser aplicadas en los procesos educativos" (p.11).

El objetivo de los profesores cuyo interés se centra en los procesos de aprendizaje de sus estudiantes, empleando nuevas herramientas tecnológicas y 10 materiales didácticos innovadores, no es, ser un experto en computación o informática, sino que mas bien, se trata de convertirse en un profesor conocedor de las ventajas y desventajas de la introducción de estas herramientas y materiales y sobre todo, lo más importante: cómo lograr enseñar, a partir de ellos.

Derivado de esta investigación, se propone trabajar en la construcción de un curso de capacitación, para el desarrollo de competencias digitales básicas, para lograr con ello, habilitar al profesorado en cuestión en compatencias de "presentación", para imparcar directa y significativamente en los procesos de aprendizaje de sus estudiantes. 


\section{REFERENCIAS BIBLIOGRÁFICAS}

Andersen, M. (9 de septiembre de 2009) Technology Skills We Should Be Teaching in College. [Mensaje en un blog]. Busynessgirl. Recuperado de: http://busynessgirl.com/technologyskills-we-should-be-teaching-in-college/

Arias, Fidias. (2004) El proyecto de investigación. Caracas: Episteme.

Cabero, J. (2007), Las necesidades de las TIC en el ámbito educativo: oportunidades, riesgos y necesidades, Universidad de Sevilla (España - UE) Recuperado de: http://investigacion.ilce.edu.mx/ tyce/45/articulo1.pdf

Cabero, J. (2010). Los retos de la integración de las TICs en los procesos educativos. Límites y posibilidades. Perspectiva Educacional, Formación de Profesores, 49 ( 1), 32-61.

Casamayor, Gregorio; La Formación on-line una mirada integral sobre e-learning, b-lerning; Ed. Grao; primera edición octubre 2008, segunda reimpresión junio 2010; España, Barcelona.

Díaz-Maroto, I., \& Cascales Martínez, A. (2015). Las TIC y las necesidades específicas de apoyo educativo: análisis de las competencias TIC en los docentes. RIED. Revista Iberoamericana de Educación a Distancia, 18 (2), 355-383.

Escuela Superior de Informática. (s.f). Prácticas de Estadística. Recuperado de: https:// www.uclm.es/profesorado/raulmmartin/ Estadistica/Practicas SPSS/ CORRELACION_CON_SPSS.pdf

Florescano, E. (1 de mayo de 1999). Para qué enseñar la historia. Nexos. Recuperado de: https://www.nexos.com.mx/?p=9250
Gobierno de la República. (2012). Plan Nacional de Desarrollo 2013-2018. Recuperado de http://www.dof.gob.mx/nota_detalle_ popup.php?codigo $=5299465$

Guzmán, I., \& Marín, R. (2011). La competencia $y$ las competencias docentes: reflexiones sobre el concepto y la evaluación. REIFOP, Revista Electrónica Interuniversitariade Formación del Profesorado, 14(1), 151-163. Recuperado de http://www. aufop.com/aufop/uploaded_files/ articulos/1301588498.pdf

Hernández, R., Bautista, D.E., y Hernández, J.J. (2015). Aprendiendo historia mediante el uso de un libro digital interactivo. En Trejo, D. y Martínez, J. (Coord.) La Historia enseñada a discusión. Retos epistemológicos y perspectivas didácticas. (pp.1024 -1041). Morelia, México: Universidad Michoacana de San Nicolás de Hidalgo.

Hernández, R., Fernández, C. y Baptista, P. (2006). Metodología de la Investigación. Cuarta Edición. Mc Graw Hill. México.

Kovachi. (s.f). Coeficiente de correlación lineal de Pearson. Recuperado de: http:// kovachi.sel.inf.uc3m.es/@api/deki/ files/141/=correlacion.pdf

Miguel, A. (2014) Las TIC y su aplicación a la enseñanza de la historia; Soria; Recuperado de: https://uvadoc.uva.es/ bitstream/10324/8378/1/TFG-O\%20 378.pdf

Organización de las Naciones Unidas para la Educación, la Ciencia y la Cultura. (2008). Estándares de competencias en TIC para docentes. Londres: Autor. Recuperado de http://eduteka.icesi.edu.co/pdfdir/ UNESCOEstandaresDocentes.pdf 
Ortega Domínguez, L., \& Reyes Sánchez, O. (2012). ¿Cómo formar competencias profesionales en el nivel superior? Revista Electrónica Educare, 16 (2), 2554.

Reig, D. (16 de septiembre 2019). Checklist de Competencias, habilidades digitales básicas. [Mensaje en un blog]. El Caparazón. Recuperado de: http:// www.dreig.eu/caparazon/2009/09/16/ checklist-competencias-habilidadesdigitales-basicas/

Reforma educativa, (2017); Recuperado de http://seduc.edomex.gob.mx/sites/ seduc.edomex.gob.mx/files/files/ Padres\%20de\%20familia/Resumen_ Ejecutivo_de_la_Reforma_Educativa. pdf Octubre 25 de 2017

Sabino, C. (2002). El Proceso de Investigación. Panapo. Venezuela.

Salinas, J., \& de Benito, B., \& Lizana, A. (2014). Competencias docentes para los nuevos escenarios de aprendizaje.Revista Interuniversitaria de Formación del Profesorado, 28 (1), 145-163. Recuperado de: https://www. redalyc.org/articulo.oa?id=27431190010

SecretaríadeEducaciónPública.(29deoctubrede 2008). Reforma Integral de la Educación Media Superior. Acuerdo 447. Diario oficial de la federación. Recuperado de http://www.sems.gob.mx/work/ models/sems/Resource/10905/1/ images/Acuerdo_447_competencias_ docentes_EMS.pdf

Vélez, R. Y Badillo, J. (2017) El pensamiento histórico como estrategia para el aprendizaje de la historia en el bachillerato. Congreso Nacional de Investigación Educativa. San Luis Potosí. Recuperado de: http://docplayer.
es/73669181-El-pensamiento-historicocomo-estrategia-para-el-aprendizajede-la-historia-en-el-bachillerato.html 